\title{
Cisplatin sensitizes human hepatocellular carcinoma cells, but not hepatocytes and mesenchymal stem cells, to TRAIL within a therapeutic window partially depending on the upregulation of DR5
}

\author{
BO ZHANG ${ }^{1}$, HONG SHAN $^{1,2}$, DAN LI $^{1}$, ZHENG-RAN LI $^{1}$, KANG-SHUN ZHU $^{1}$, \\ ZAI-BO JIANG $^{1}$ and MING-SHENG HUANG ${ }^{1}$
}

\author{
${ }^{1}$ Molecular Imaging Laboratory, Department of Radiology, Third Affiliated Hospital of Sun Yat-sen University; \\ ${ }^{2}$ Interventional Radiology Institute of Sun Yat-sen University, Guangzhou 510630, P.R. China
}

Received August 26, 2010; Accepted October 4, 2010

DOI: $10.3892 / o r .2010 .1084$

\begin{abstract}
Tumor necrosis factor (TNF)-related apoptosisinducing ligand (TRAIL) is a member of the TNF family of cytokines and has been shown to induce cell apoptosis in many types of tumors, but not in normal cells. This tumorselective property has made TRAIL a promising approach for the development of cancer therapy. However, hepatocellular carcinoma (HCC) cells display a striking resistance to TRAIL. Although some chemotherapeutic agents can overcome this resistance, safety issues remain a concern because the combination of these agents and TRAIL has been reported to induce toxicity in normal hepatocytes. In this study, we examined whether cisplatin could reverse TRAIL resistance in HCC cells with different p53 status and evaluated the toxicity of combination TRAIL and cisplatin to normal hepatocytes and mesenchymal stem cells (MSCs). We observed that cisplatin could efficiently sensitize HCC cells, but not hepatocytes and MSCs to TRAIL-induced apoptosis within a wide therapeutic window. The apoptosis of HCC cells only partially depended on the upregulation of DR5 and the status of $\mathrm{p} 53$. In addition, we provide favorable evidence supporting the feasibility of the combination of chemotherapy and MSCs transduced with TRAIL.
\end{abstract}

\section{Introduction}

Hepatocellular carcinoma (HCC) is the fifth most common cancer worldwide and the third-leading cause of death. HCC

Correspondence to: Dr Hong Shan, Department of Radiology, Third Affiliated Hospital of Sun Yat-sen University, Guangzhou 510630, P.R. China

E-mail: shanhong5@gmail.com

Key words: hepatocellular carcinoma, TRAIL, cisplatin, apoptosis, death receptor, p53 is often diagnosed at an advanced stage. Despite a variety of treatment procedures including surgical resection, transcatheter arterial chemoembolization, percutaneous radiofrequency ablation or injection of ethanol, and liver transplantation, the prognosis of HCC patients is far from being satisfactory with a dismal 5-year survival rate for its invasiveness and high resistance to conventional chemotherapy (1-3). Thus, novel therapeutic strategies are required to reduce drug dosage, enhance the therapy efficacy, and to diminish toxicity to normal cells.

Tumor necrosis factor (TNF)-related apoptosis-inducing ligand (TRAIL), the apoptosis-inducing member of the TNF super family, can initiate apoptosis in a variety of human cancer cell lines through the activation of their death receptors. Four major TRAIL receptors have been identified: TRAILR1 (death receptor, DR4) and TRAIL-R2 (DR5) are receptors with fully functional cytoplasmic death domains. On the other hand, TRAIL-R3 (Decoy receptor, DcR1) and TRAIL-R4 (DcR2) have either an obliterated (DcR1) or a truncated (DcR2) death domain, thus TRAIL signaling cascade can not be completed. These decoy receptors have been proposed to competitively inhibit TRAIL-induced apoptosis by acting as nonfunctional receptors $(4,5)$.

However, $\sim 60 \%$ of tumor cells were resistant to TRAIL, which severely limits the application of TRAIL in cancer treatment. Though some chemotherapeutic agents or radiation can sensitize HCC cells, conflicting results have been published with regard to the sensitivity of normal human hepatocytes to TRAIL exposure (6). In addition, increased expression of DcR1/DcR2 and decreased DR4/DR5 expression correlates with TRAIL resistance, but the expression levels of TRAIL receptors do not correlate with TRAIL sensitivity $(7,8)$. Therefore, it is important to understand the mechanisms involved in resistance and sensitization to TRAIL-induced apoptosis.

Although TRAIL has been shown to be capable of inducing apoptosis in tumor cells of diverse origin, only a few studies on HCC cells with different p53 status, normal hepatocytes and mesenchymal stem cells have been published (6,9-11). In our study, we demonstrated that the TRAIL resistance of 
HCC cell lines with different p53 status could be reversed by cisplatin, while normal hepatocytes and MSCs are not affected significantly. Moreover, the mechanism of TRAIL sensitization by cisplatin in HCC cells was also analyzed.

\section{Materials and methods}

Cell culture. HCC cell lines Bel-7402 (p53 wild-type) (12,13), Huh 7 (p53 mutant) (14), Hep3B (p53 defective) (14), hepatocytes L-02 and mesenchymal stem cells (MSCs) were grown in Dulbecco's modified Eagle's medium with $10 \%$ fetal bovine serum and penicillin $(100 \mathrm{U} / \mathrm{ml}) /$ streptomycin $(100 \mu \mathrm{g} / \mathrm{ml})$ and were maintained at $37^{\circ} \mathrm{C}$ in a humidified atmosphere with $5 \% \mathrm{CO}_{2}$.

Antibodies and reagents. For fluorescence-activated cell sorting (FACS) analysis, Mouse IgG1 Isotype Control-PE (IC002P), IgG2B Isotype Control-PE (IC0041P), TRAIL R1 (DR4)-PE (FAB347P), TRAIL R2 (DR5)-PE (FAB6311P), TRAIL R3 (DcR1)-PE (FAB6302P) and TRAIL R4 (DcR2)PE (FAB633P) were purchased from R\&D Systems. For Western blots, the following primary antibodies were used: anti-caspase-8 (CST, \#9746), caspase-9 (CST, \#9502), caspase-3 (CST, \#9662) and B-actin antibody (CST, \#4967). HRP-conjugated secondary antibodies were also obtained from CST (\#7076 and \#7074). Soluble recombinant human TRAIL was purchased from PeproTech. Cisplatin was obtained from Sigma.

Cell viability assay. Cell viability assay was performed as Miyoshi N as described (15). In brief, HCC cells $(20,000$ per well), hepatocytes $(20,000$ per well) and MSCs (5,000 cells per well) were seeded in a 96-well plate. After an overnight incubation, they were treated with $100 \mu \mathrm{l}$ medium containing cisplatin $(0,2.5$ and $5.0 \mu \mathrm{g} / \mathrm{ml})$ and TRAIL $(0,50,100,200 \mathrm{ng} /$ $\mathrm{ml}$ ) for $24 \mathrm{~h}$. Then, $10 \mu \mathrm{l}$ of a Cell Counting kit-8 (Dojindo Laboratories, Japan) solution was added to each well. After incubation at $37^{\circ} \mathrm{C}$ for $2 \mathrm{~h}$ in a humidified $\mathrm{CO}_{2}$ incubator, absorbance of each well was monitored with a microplate reader (ELX-800, Bio-Tek) using $450 \mathrm{~nm}$ as the primary wavelength (630 $\mathrm{nm}$ as the reference wavelength).

FACS analysis of apoptosis. The sub-G1 analysis after propidium iodide (PI) staining was employed to assess the apoptosis. After treatment with TRAIL (100 ng/ml) and/or cisplatin $(5.0 \mu \mathrm{g} / \mathrm{ml})$ for $24 \mathrm{~h}, \mathrm{HCC}$ cells, hepatocytes and MSCs were harvested. The cells were washed twice with cold PBS, resuspended in $1 \mathrm{ml}$ of $70 \%$ ethanol, and kept overnight. They were subsequently rinsed twice with PBS and incubated with $100 \mu 1 \mathrm{RNase}(10 \mathrm{mg} / \mathrm{ml})$ at $37^{\circ} \mathrm{C}$ for $30 \mathrm{~min}$. Finally, the cells were stained with PI $(0.5 \mu \mathrm{g} / \mathrm{ml}$, Sigma) for $30 \mathrm{~min}$ in the dark at $4^{\circ} \mathrm{C}$. Distribution of the cell cycle and the rate of apoptosis were determined on a BD LSRII cytometer (BD Biosciences).

FACS analysis of TRAIL receptors. HCC cells, hepatocytes and MSCs (controls or treated with cisplatin at $5.0 \mu \mathrm{g} / \mathrm{ml}$ for $24 \mathrm{~h}$ ) were detached non-enzymatically with citric saline buffer ( $0.135 \mathrm{M}$ potassium chloride, $0.015 \mathrm{M}$ sodium citrate) for 5-8 $\mathrm{min}$ at $37^{\circ} \mathrm{C}$. FACS analysis of TRAIL receptors was performed according to the manufacturer's protocol $(R \& D$ Systems). In brief, cells were centrifuged and washed three times in an isotonic PBS buffer (supplemented with $0.5 \%$ BSA), and were incubated with IgG1 Isotype, IgG2B Isotype and TRAIL R1-R4 antibodies (10 $\mu 1 / 100,000$ cells) respectively, for $40 \mathrm{~min}$ at $4^{\circ} \mathrm{C}$. Excess unbound antibodies were removed by washing twice with PBS before submission for FACS. The levels of receptor expression were quantified by the PE mean fluorescence intensity index (ratio of PE mean fluorescence intensity of cells incubated with antireceptor antibodies and the background PE mean fluorescence intensity in cells incubated with IgG isotype control) and the percentages of cells gated positive for receptor expression.

Western blot analysis. Preparation of cell lysates and immunoblotting were done as described before (9). In brief, protein supernatants were denatured and $20 \mu \mathrm{g}$ of protein were separated on a gradient $10 \%$ to $12 \%$ SDS-PAGE and transferred onto Polyvinylidene-Fluoride (PVDF) membranes. The membranes were probed with primary antibodies against caspase $-8,-9,-3$ and $B$-actin, which were diluted at 1:1,000 with $3 \%$ bovine serum albumin $(\mathrm{pH} \mathrm{7.4).} \mathrm{The} \mathrm{blots} \mathrm{were}$ developed using an HRP-conjugated secondary antibody and visualized with chemiluminescent substrate (Thermo, SuperSignal West Pico 34079). Relative optical densities of protein bands were quantitated by using the Bio-Rad Quantity One version 4.6.2.

Statistical analysis. Results were expressed as mean \pm standard deviation (SD). Differences between groups were compared using two-tailed Student's t-tests. $\mathrm{P}<0.05$ was considered statistically significant.

\section{Results}

Combined use of cisplatin and TRAIL enhanced the inhibition of cell proliferation in HCC cells, but not in hepatocytes and MSCs within a therapeutic window. We examined the effect of TRAIL and cisplatin on the viability of HCC cells with different p53 status. TRAIL or cisplatin alone failed to affect the viability of HCC cells significantly when $200 \mathrm{ng} / \mathrm{ml}$ of TRAIL or $5 \mu \mathrm{g} / \mathrm{ml}$ of cisplatin was added to the culture. While even in the presence of low concentrations of cisplatin (2.5 or $5.0 \mu \mathrm{g} / \mathrm{ml})$, TRAIL dose-dependently reduced the cell viability (Fig. 1A-C). The viability of hepatocytes and MSCs were rarely affected by the combination of $200 \mathrm{ng} / \mathrm{ml}$ TRAIL and $5 \mu \mathrm{g} / \mathrm{ml}$ cisplatin (Fig. 1D and E). In addition, up to $20 \mu \mathrm{g} / \mathrm{ml}$ cisplatin did not sensitize hepatocytes even for $1,000 \mathrm{ng} / \mathrm{ml}$ TRAIL (Fig. 1F), which suggested a wide therapeutic window for the combination of cisplatin and TRAIL.

Cisplatin-induced upregulation of DR5 and DcR2 in HCC cells, but not in hepatocytes and MSCs. TRAIL receptors on the cell surface were investigated by FACS. All HCC cells express DR5 and DcR2, however, DR4 and DcR1 did not appear to be expressed in these HCC cells, except Hep3B which express low levels of DR4. After $24 \mathrm{~h}$ of treatment with $5.0 \mu \mathrm{g} / \mathrm{ml}$ cisplatin, although there was no change in the expression of the DR4 and DcR1, the expression of DR5 and DcR2 was increased in Bel-7402 (2.01-fold and 2.28-fold), 

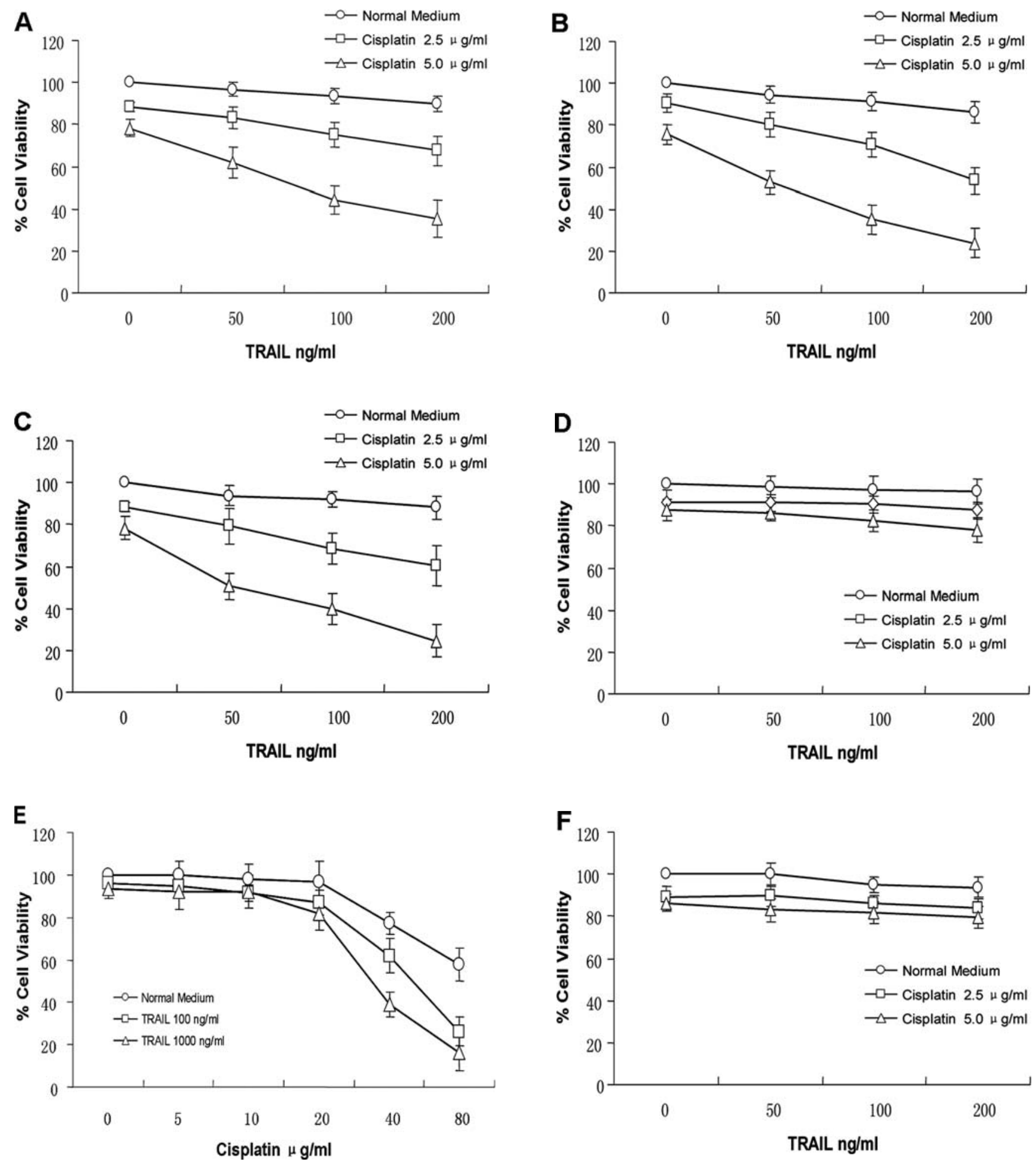

Figure 1. Enhanced suppression effects of HCC cells proliferation by combination of TRAIL with cisplatin. Three HCC cell lines, Bel-7402 (A), Huh 7 (B), Hep3B (C), hepatocytes L-02 (D and E) and MSCs (F) were treated with different concentrations of TRAIL and cisplatin for 24 h. Cell viability was determined by CCK-8 assay. Each point represents the mean \pm SD of three independent experiments.

Huh 7 (1.18-fold and 2.67-fold) and Hep3B (1.28-fold and 1.27-fold). On the other hand, only mild expression of DcR2 in L-02 and that of DR5 and DcR1 in MSCs were observed respectively. Though DR5 in L-02 and MSCs was upregulated in some independent experiments, no significant differences between treatment and control groups was observed in these normal cells (Fig. 2).
TRAIL selectively induced apoptosis in HCC cells after sensitization with cisplatin, which partially depended on the upregulation of DR5 and status of p53, whereas hepatocytes and MSCs remained TRAIL resistant. The monotherapy with either cisplatin $(5.0 \mu \mathrm{g} / \mathrm{ml})$ or TRAIL $(100 \mathrm{ng} / \mathrm{ml})$ induced a faint apoptotic rate in Bel-7402 $(<6 \%)$, Huh $7(<3 \%)$ and Hep3B $(<13 \%)$ cells. However, cotreatment with TRAIL 
TRAIL-R1 (DR4) TRAIL-R2 (DR5) TRAIL-R3 (DcR1) TRAIL-R4 (DcR2)
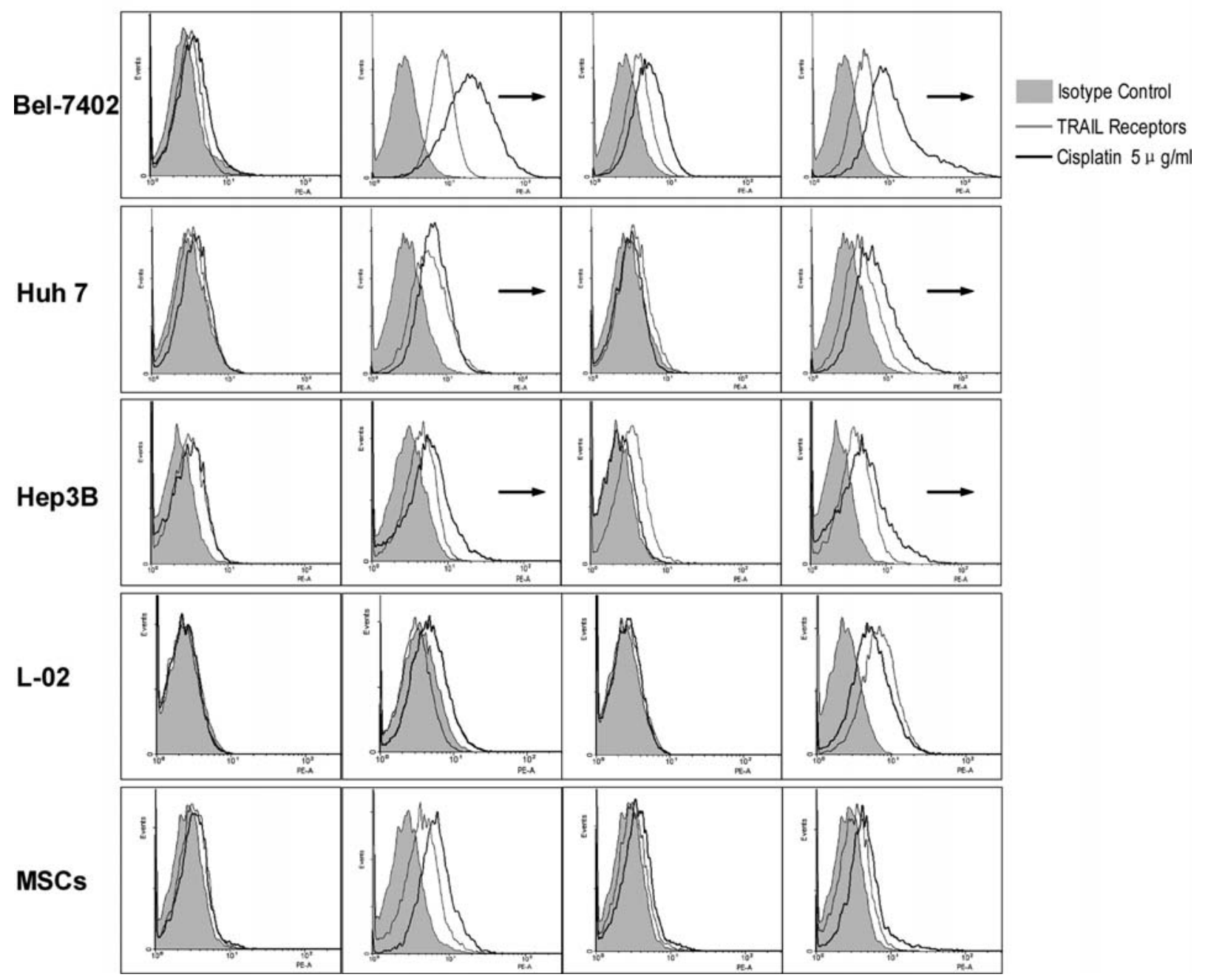

Figure 2. Flow cytometric analysis of surface DR4, DR5, DcR1 and DcR2 expression. HCC cells, hepatocytes and MSCs were treated with cisplatin $(5.0 \mu \mathrm{g} / \mathrm{ml})$ for $24 \mathrm{~h}$. In HCC cell lines, the expression of DR5 and DcR2 were upregulated, while those of DR4 and DcR1 were not significantly altered. The TRAIL receptors in L-02 and MSCs were more variable. Despite DR5 in L-02 and MSCs was upregulated in this independent experiment, this difference was not statistically significant. The results are shown from three independent experiments.

and cisplatin strongly induced apoptosis in these HCC cells, indicating that the sensitivity of these cells to TRAIL was remarkably enhanced by cisplatin (Fig. 3A and B). However, the apoptotic rates induced by TRAIL/cisplatin in Bel-7402 (14.91 $\pm 4.31 \%)$, Huh 7 (37.66 $\pm 6.33 \%)$ and Hep3B $(45.38 \pm 10.58 \%)$ (Fig. 4A) were not in proportion to the upregulation of DR5 (2.01-, 1.18- and 1.28-fold increase in Bel-7402, Huh 7 and Hep3B, respectively) (Fig. 4B). Additionally, statistical analysis revealed Huh 7 (p53 mutant) and Hep3B (p53 defective) were more sensitive than Bel-7402 (p53 wild-type) to TRAIL/cisplatin combination (Fig. 4A).

Though the same concentration of cisplatin showed a very mild toxicity to hepatocytes and MSCs (apoptotic rates $<5.0 \%$ ), these normal cells all represented a high resistance to TRAIL alone or TRAIL/cisplatin combination, suggesting that cisplatin and TRAIL do not act synergistically in these normal cells (Fig. 4A).

Synergistic induction of apoptosis in HCC cells exposed to TRAIL/Cisplatin involve activation of both the extrinsic and the intrinsic pathways. To confirm the apoptotic pathway that might be involved, we detected the activation of the extrinsic (caspase-8) and the intrinsic (caspase-9) signaling cascades. Though cisplatin alone led to the faint cleavage of caspase-3, -8 and -9 in Bel-7402 and Hep3B, their cleaved forms were more obvious with the co-treatment of cisplatin and TRAIL in Bel-7402, Huh 7 and Hep3B (Fig. 5). In addition, the cleaved fragments of caspase- 8 of Huh 7 seemed weaker than those in Bel-7402 and Hep3B, however, the cleaved fragments of caspase- 9 were the opposite. In contrast, even $1,000 \mathrm{ng} / \mathrm{ml}$ TRAIL and $20 \mu \mathrm{g} / \mathrm{ml}$ cisplatin did not induce the activation of caspase- $8,-9$ and -3 in L-02 and MSCs.

\section{Discussion}

Apoptosis (programmed cell death) is the cell intrinsic death program that is involved in the regulation of many physiological and pathological processes and that is evolutionary highly conserved (16). Since tissue homeostasis is the result of a subtle balance between proliferation and cell death, 

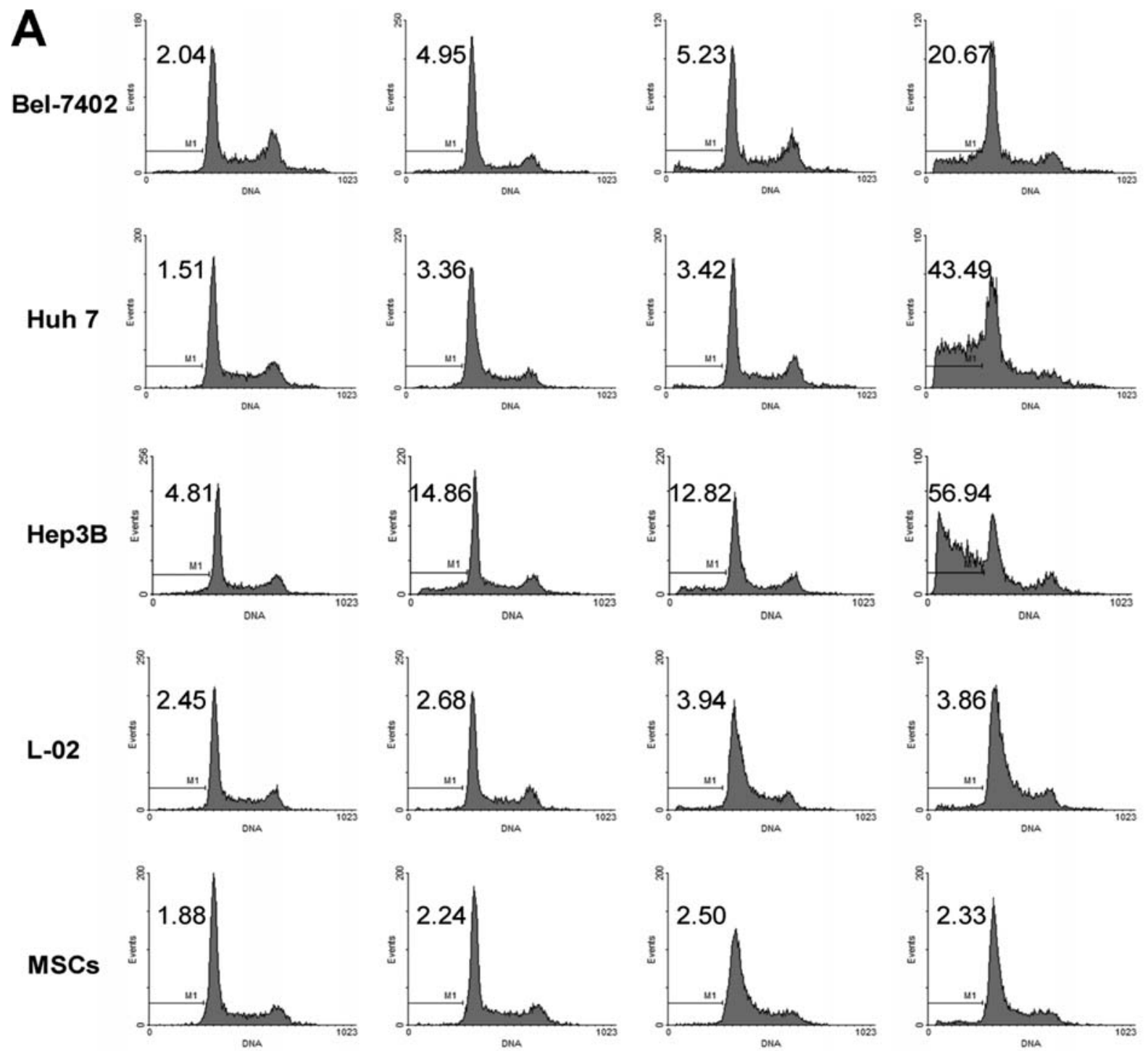

Normal Medium

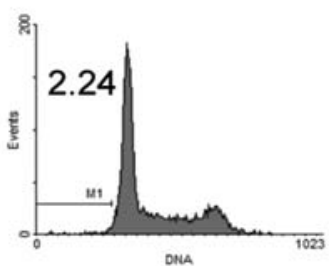

TRAIL

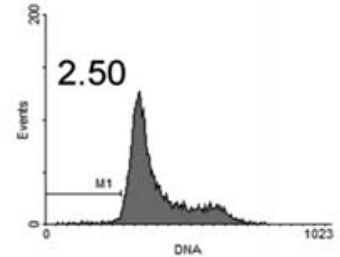

Cisplatin

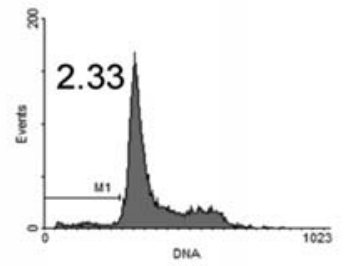

TRAIL+Cisplatin

B

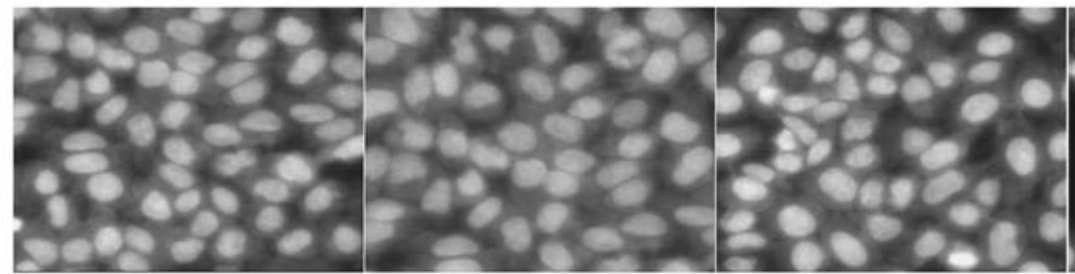

Normal Medium

TRAIL

Cisplatin

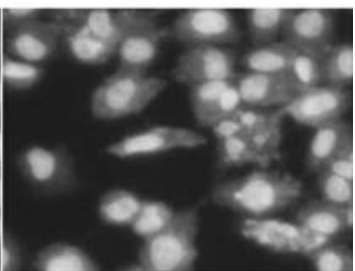

TRAIL+Cisplatin

Figure 3. HCC cells, hepatocytes and MSCs were treated with TRAIL (100 ng/ml), cisplatin ( $5.0 \mu \mathrm{g} / \mathrm{ml})$, or the combination of both reagents for $24 \mathrm{~h}$. (A) Enhanced induction of apoptosis by the combination treatment was clearly observed in Bel-7402, Huh 7 and Hep3B, however L-02 and MSCs were resistant to the combination treatment and did not undergo apoptosis. (B) Bel-7402 cell nuclei were stained with Hoechst 33258.

evasion of apoptosis may contribute to carcinogenesis, tumor progression as well as treatment resistance. Most current anticancer therapies including chemotherapy, radio- and immunotherapy primarily act by activating cell death pathways including apoptosis in cancer cells (17). Apoptosis is coordinated and fine tuned by a set of proteins that are either regulators or executors of the process. One of the features of human cancers is the intrinsic or acquired resistance to apoptosis. Therefore, a better understanding of the molecular mechanisms that induce tumor resistance to apoptosis is expected to provide a molecular basis for the design of new strategies to reverse apoptosis resistance of human cancers.

Generally, there are two major apoptotic pathways, namely the extrinsic death receptor and intrinsic mitochondrial pathways. Binding of TRAIL to DR4 or DR5 leads to receptor oligomerization on the cell membrane and initiation of apoptosis through the recruitment of an adaptor protein Fasassociated death domain (FADD). FADD functions as a 
A

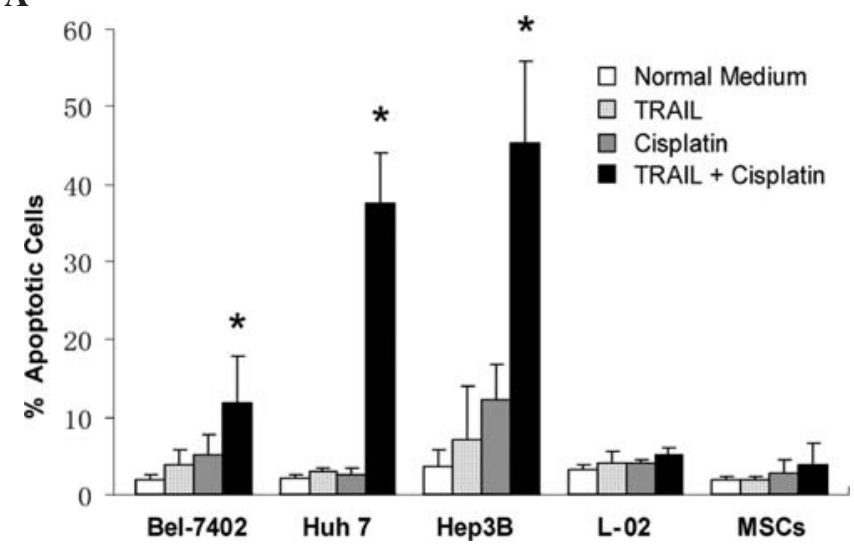

B

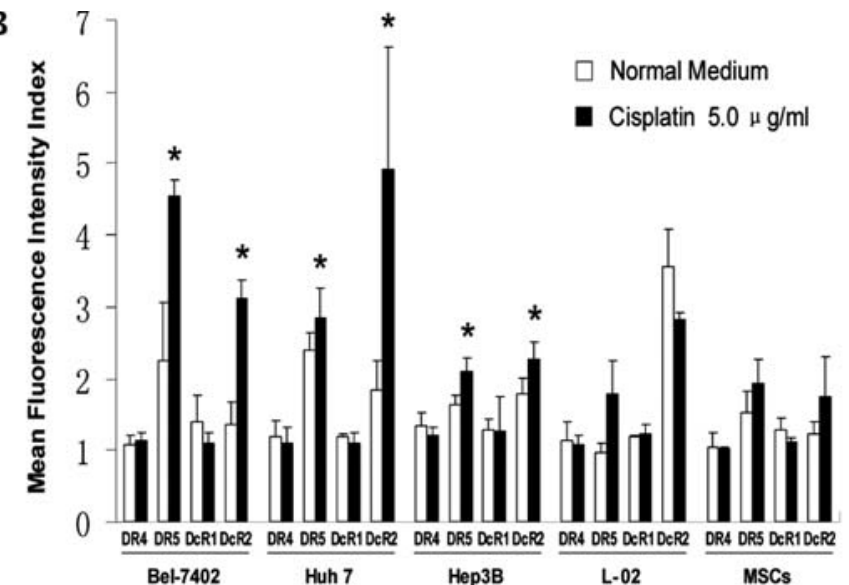

Figure 4. The apoptosis induced by TRAIL/cisplatin in HCC cells partially depended on the upregulation of DR5. After $24 \mathrm{~h}$ of treatment with TRAIL (100 ng/ml) and/or cisplatin $(5.0 \mu \mathrm{g} / \mathrm{ml})$, the apoptotic rates in Bel-7402, Huh 7 and Hep3B (A) were not quite in proportion to the upregulation of DR5 (B). In addition, Huh 7 and Hep3B were more sensitive than Bel-7402 to TRAIL/cisplatin combination (A). Experiments were repeated at least 3 times. Data are shown as the mean $\pm \mathrm{SD}$. Asterisk indicates $\mathrm{p}<0.05$.

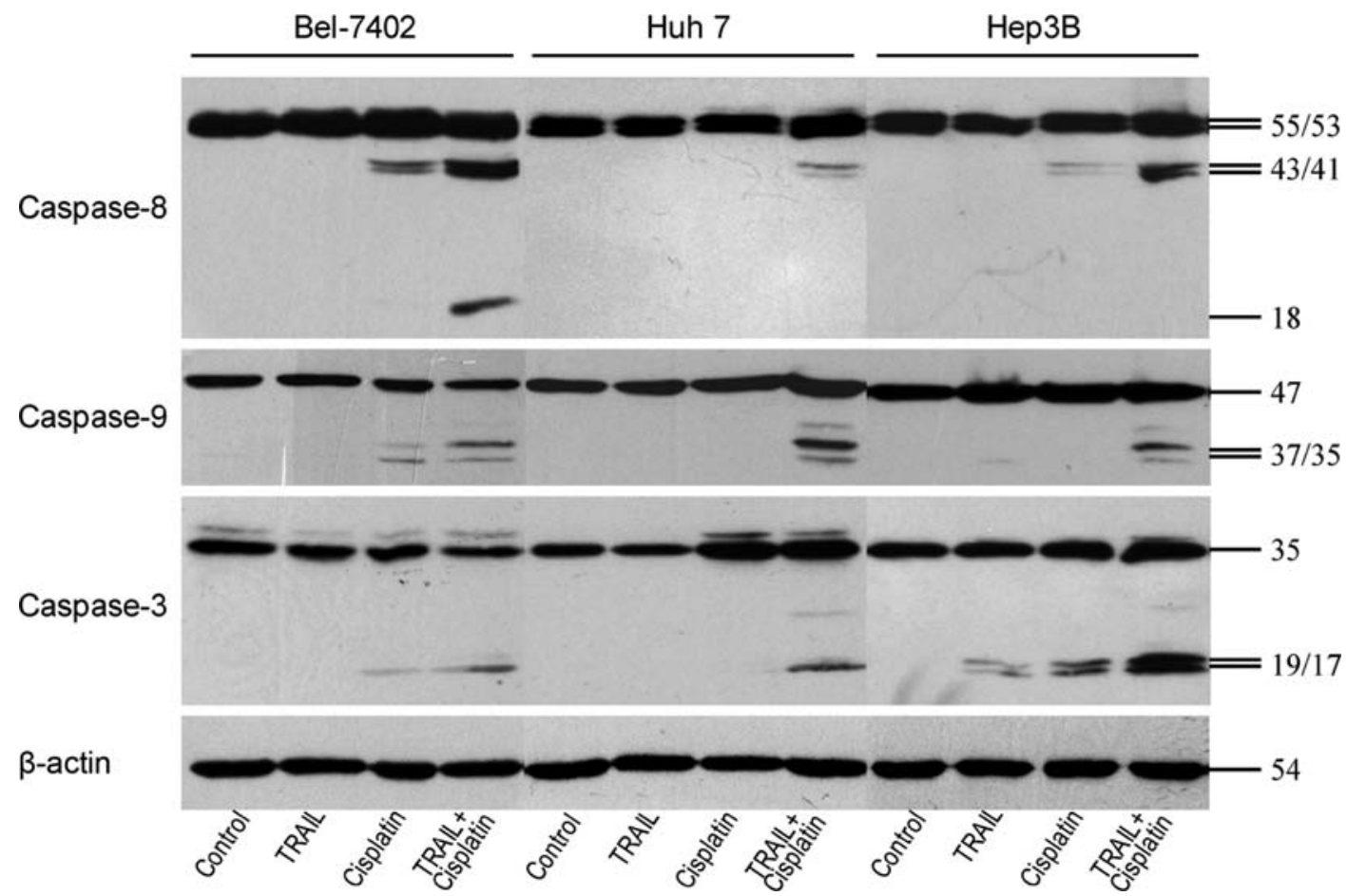

Figure 5. Changes in caspase- $8,-9,-3$ protein expression. In the combination of TRAIL (100 $\mathrm{ng} / \mathrm{ml})$ and cisplatin (5.0 $\mu \mathrm{g} / \mathrm{ml})$ for $24 \mathrm{~h}$, procaspases in these HCC cells were significantly activated with an apparent increase in the cleaved caspase expression. Molecular weights (in kDa), and cleaved fragments are indicated. One of three comparable experiments is shown.

molecular bridge to membrane proximal caspase- 8 , a protease inciting the follow-up cell death cascades (18). Once caspase-8 is activated via autocatalysis, the activated fragments stimulate apoptosis by the two fundamentally distinct signaling cascades (19). For the extrinsic pathway, activated caspase-8 directly leads to activation of downstream effector caspases-3, and activated caspase- 3 in turn cleaves numerous cellular proteins, ultimately resulting in an irreversible commitment of cells to undergo apoptosis $(20,21)$.

In contrast, the intrinsic pathway is controlled by mitochondria. Activation of this pathway involved the cleavage of $\mathrm{BH} 3$ interacting death domain and mitochondrial outer membrane permeabilization, resulting in the release of cytochrome c from mitochondria, which sequentially activates caspase-9 and -3. Shamimi-Noori S et al revealed that cisplatin/TRAIL-induced cytotoxicity of thoracic cancer cells was completely abrogated either by the selective caspase- 9 inhibitor or by transient knockdown of caspase-9 by siRNA, indicating that this apoptotic process was caspase-mediated and mitochondria-dependent (22). In the present study, we investigated the signaling cascades of apoptosis after the combination of TRAIL and cisplatin by Western blotting. These results suggest that the synergistic effect of TRAIL and cisplatin is achieved through both the extrinsic (caspase-8) 
and intrinsic (caspase-9) pathways in all three HCC cell lines. Furthermore, the activation of caspase- 9 in Huh 7 seemed more obvious than that of Bel-7402 and Hep3B.

p53 is the most commonly mutated tumor suppressor gene involved in pathogenesis of hepatocellular carcinoma. When the DNA is damaged in the cells (genetic code), intracellular concentrations of p53 are greatly increased. Protein product of p53 gene is involved in cell cycle arrest and cellular apoptosis. If p53 itself has mutated and no longer works properly, proliferation of damaged cells can promote tumor formation as well as progression $(23,24)$. However, it is not yet completely understood how the activation of p53 initiates apoptosis. In addition, the role of p53 in the chemotherapeutic resistance of HCC cells is still unclear. As a downstream gene of the tumor suppressor p53 gene, DR5 has been demonstrated to be induced by DNA-damaging agents in a p53-dependent fashion (25), and its transcription is directly transactivated by p53 through an intronic sequencespecific p53BS. It has been confirmed that mutant p53 inhibits p53-dependent transactivation of DR5 (26). In addition, DR4 is a DNA damage-inducible, p53-regulated gene, although the p53 binding site in its 5'-flanking region was not determined (27). Interestingly, the expression of DcR1 and DcR2 is also induced by p53 (28). It has been demonstrated that p53 regulates DcR1 expression through an intronic p53 binding site (29).

Our current study suggested that only DR5 and DcR2 were expressed in all investigated HCC cell lines with different p53 status, whereas DR4 and DcR1 were not expressed obviously, even in Bel-7402 with wild-type p53. When these HCC cells were cultured with cisplatin, DR5 was increased 2.01-fold in Bel-7402 compared with that of untreated cells. While in Huh 7 and Hep3B, the values of DR5 upregulation were only 1.18 -fold and 1.28 -fold, which suggested the increase of DR5 might partially depend on p53. We found that in p53-mutate (Huh 7) and -defective (Hep3B) settings, cisplatin dramatically sensitizes tumor cells to apoptosis induced by TRAIL. Though the combination of TRAIL and cisplatin resulted in a synergistic effect in Bel-7402, the apoptotic rates of Bel-7402 were much less than those of Huh 7 and Hep3B, which indicates that the apoptotic rates induced by TRAIL were not quite in proportion to the upregulation of DR5. Briefly, the apoptosis induced by TRAIL and cisplatin only partially depended on the status of p53, and the role of p53 in the apoptosis of HCC cells might be very complex and controversial, and needs further study in cells with p53 knockdown.

After an initial debate on potential liver toxicity of TRAIL (6), a consensus has been reached that only highly aggregated recombinant forms of TRAIL (e.g. His-TRAIL) are toxic to freshly isolated primary human hepatocytes at day 1 of in vitro culture, and the other forms of TRAIL alone do not show significant hepatocyte cytotoxicity $(9,30,31)$, which is consistent with our results. In TRAIL-sensitive human hepatocytes in vitro, the apoptosis induced by TRAIL could be prevented by DR4/DR5 blocking antibodies (9). Thus, pro-inflammatory conditions, viral hepatitis and super high-dose of chemotherapeutic drugs have been shown to sensitize human and murine hepatocytes for TRAIL by upregulation of DR4 and/or DR5 $(32,33)$. Our study confirmed that $5 \mu \mathrm{g} / \mathrm{ml}$ cisplatin-treated hepatocytes did not increase DR4 and DR5 expression significantly. Even treated with $20 \mu \mathrm{g} / \mathrm{ml}$ cisplatin and 1,000 $\mathrm{ng} / \mathrm{ml}$ TRAIL, hepatocytes remained TRAIL-resistant, which suggested a wide therapeutic window for the combination of cisplatin and TRAIL in treatment of HCC.

Human multipotent mesenchymal stem cells (MSCs) are able to self-renew, proliferate, and differentiate into a variety of cell types (34). MSCs have also been demonstrated to migrate towards tumors in response to chemokines produced by tumor cells $(10,35)$. This MSC tropism offers numerous therapeutic applications for tumors. In addition, genetically modified MSCs have been shown to have high metabolic activity and strong expression of transgenes in vitro and in vivo, and retain their 'stem-like' characteristics and home to tumors (11). Therefore, it is urgent to investigate the property of MSCs under the treatment of TRAIL and chemotherapeutic agents. Our study demonstrated that MSCs expressed both DR5 and DcR2, while DR4 and DcR1 were not detected by FACS analysis. After treated with cisplatin, neither upregulation nor downregulation of these receptors was observed in MSCs. MSCs represented a high resistance to TRAIL alone or TRAIL/cisplatin combination. Hence, even under cisplatin treatment, MSCs could still be used as a gene vector to express TRAIL.

In summary, we have shown that HCC cell lines were all high resistant to TRAIL treatment alone, though DR5 on the cell surface were prevalent in HCC cells. However, this resistance was significantly reversed by cisplatin, and the upregulation of DR5 might contribute to this reversal, although the upregulated DR5 was not in proportion to the apoptotic rates induced by TRAIL. p53 played a positive regulatory role in DR5 upregulation by cisplatin, while p53 seemed to prevent HCC cells from apoptosis induced by TRAIL and cisplatin, thus the physiological functions of p53 have not yet been elucidated and need a deeper insight. In contrast to HCC cells, hepatocytes and MSCs showed a high resistance to TRAIL alone or TRAIL/cisplatin combination. Our results imply that TRAIL in combination with chemotherapeutic agents might be a promising option for the treatment of human HCC without obvious hepatocyte toxicity and other normal cells within a wide therapeutic window. In addition, our data could provide favorable evidence supporting the feasibility of the combination of MSCs transduced with TRAIL gene and chemotherapy in the treatment of TRAIL-resistant human cancer.

\section{Acknowledgements}

This study was supported by Team Project of Natural Science Foundation of Guangdong Province (05200177), National Natural Science Foundation of China (30770628), Science Project Foundation of Guangdong Province (No. 2008A030201004) and Natural Science Foundation of Guangdong Province (8151008901000066), P.R. China.

\section{References}

1. Roberts LR: Sorafenib in liver cancer - just the beginning. N Engl J Med 359: 420-422, 2008 
2. Llovet JM, Burroughs A and Bruix J: Hepatocellular carcinoma. Lancet 362: 1907-1917, 2003.

3. Aravalli RN, Steer CJ and Cressman EN: Molecular mechanisms of hepatocellular carcinoma. Hepatology 48: 2047-2063, 2008

4. Wiley SR, Schooley K, Smolak PJ, et al: Identification and characterization of a new member of the TNF family that induces apoptosis. Immunity 3: 673-682, 1995.

5. Walczak H, Miller RE, Ariail K, et al: Tumoricidal activity of tumor necrosis factor-related apoptosis-inducing ligand in vivo. Nat Med 5: 157-163, 1999.

6. Jo M, Kim TH, Seol DW, Esplen JE, Dorko K, Billiar TR and Strom SC: Apoptosis induced in normal human hepatocytes by tumor necrosis factor-related apoptosis-inducing ligand. Nat Med 6: 564-567, 2000.

7. Hopkins-Donaldson S, Ziegler A, Kurtz S, et al: Silencing of death receptor and caspase-8 expression in small cell lung carcinoma cell lines and tumors by DNA methylation. Cell Death Differ 10: 356-364. 2003.

8. Hietakangas V, Poukkula M, Heiskanen KM, Karvinen JT, Sistonen L and Eriksson JE: Erythroid differentiation sensitizes K562 leukemia cells to TRAIL-induced apoptosis by downregulation of c-FLIP. Mol Cell Biol 23: 1278-1291, 2003.

9. Ganten TM, Koschny R, Sykora J, et al: Preclinical differentiation between apparently safe and potentially hepatotoxic applications of TRAIL either alone or in combination with chemotherapeutic drugs. Clin Cancer Res 12: 2640-2646, 2006.

10. Mohr A, Lyons M, Deedigan L, et al: Mesenchymal stem cells expressing TRAIL lead to tumour growth inhibition in an experimental lung cancer model. J Cell Mol Med 12: 2628-2643, 2008.

11. Secchiero P, Melloni E, Corallini F, et al: Tumor necrosis factorrelated apoptosis-inducing ligand promotes migration of human bone marrow multipotent stromal cells. Stem Cells 26: 2955-2963, 2008.

12. Shi M, Wang FS and Wu ZZ: Synergetic anticancer effect of combined quercetin and recombinant adenoviral vector expressing human wild-type p53, GM-CSF and B7-1 genes on hepatocellular carcinoma cells in vitro. World J Gastroenterol 9: 73-78, 2003.

13. Liu TG, Yin JQ, Shang BY, et al: Silencing of hdm2 oncogene by siRNA inhibits p53-dependent human breast cancer. Cancer Gene Ther 11: 748-756, 2004.

14. Müller M, Wilder S, Bannasch D, et al: p53 activates the CD95 (APO-1/Fas) gene in response to DNA damage by anticancer drugs. J Exp Med 188: 2033-2045, 1998.

15. Miyoshi N, Oubrahim H, Chock PB and Stadtman ER: Agedependent cell death and the role of ATP in hydrogen peroxideinduced apoptosis and necrosis. Proc Natl Acad Sci USA 103: 1727-1731, 2006.

16. Hengartner MO: The biochemistry of apoptosis. Nature 407: 770-776, 2000.

17. Fulda S: Tumor resistance to apoptosis. Int J Cancer 124: 511-515, 2009.

18. Johnstone RW, Frew AJ and Smyth MJ: The TRAIL apoptotic pathway in cancer onset, progression and therapy. Nat Rev Cancer 8: 782-798, 2008

19. Kroemer G, Galluzzi L and Brenner C: Mitochondrial membrane permeabilization in cell death. Physiol Rev 87: 99-163, 2007.

20. Falschlehner C, Emmerich $\mathrm{CH}$, Gerlach B and Walczak $\mathrm{H}$ : TRAIL signalling: decisions between life and death. Int J Biochem Cell Biol 39: 1462-1475, 2007.
21. Reuter S, Eifes S, Dicato M, Aggarwal BB and Diederich M: Modulation of anti-apoptotic and survival pathways by curcumin as a strategy to induce apoptosis in cancer cells. Biochem Pharmacol 76: 1340-1351, 2008.

22. Shamimi-Noori S, Yeow WS, Ziauddin MF, et al: Cisplatin enhances the antitumor effect of tumor necrosis factor-related apoptosis-inducing ligand gene therapy via recruitment of the mitochondria-dependent death signaling pathway. Cancer Gene Ther 15: 356-370, 2008.

23. Giono LE and Manfredi JJ: The p53 tumor suppressor participates in multiple cell cycle checkpoints. J Cell Physiol 209: 13-20, 2006.

24. Halaby MJ and Yang DQ: p53 translational control: a new facet of $\mathrm{p} 53$ regulation and its implication for tumorigenesis and cancer therapeutics. Gene 395: 1-7, 2007.

25. Wu GS, Burns TF, McDonald ER III, et al: KILLER/DR5 is a DNA damage-inducible p53-regulated death receptor gene. Nat Genet 17: 141-143, 1997.

26. Takimoto R and El-Deiry WS: Wild-type p53 transactivates the KILLER/DR5 gene through an intronic sequence-specific DNA-binding site. Oncogene 19: 1735-1743, 2000.

27. Guan B, Yue P, Clayman GL and Sun SY: Evidence that the death receptor DR4 is a DNA damage-inducible, p53-regulated gene. J Cell Physiol 188: 98-105, 2001.

28. Meng RD, McDonald ER III, Sheikh MS, Fornace AJ Jr and El-Deiry WS: The TRAIL decoy receptor TRUNDD (DcR2, TRAIL-R4) is induced by adenovirus-p53 overexpression and can delay TRAIL-, p53-, and KILLER/DR5-dependent colon cancer apoptosis. Mol Ther 1: 130-144, 2000.

29. Ruiz de Almodóvar C, Ruiz-Ruiz C, Rodríguez A, Ortiz-Ferrón G, Redondo JM and López-Rivas A: Tumor necrosis factor-related apoptosis-inducing ligand (TRAIL) decoy receptor TRAIL-R3 is up-regulated by p53 in breast tumor cells through a mechanism involving an intronic p53-binding site. J Biol Chem 279: 4093-4101, 2004.

30. Lawrence D, Shahrokh Z, Marsters S, et al: Differential hepatocyte toxicity of recombinant Apo2L/TRAIL versions. Nat Med 7: 383-385, 2001.

31. Ichikawa K, Liu W, Zhao L, et al: Tumoricidal activity of a novel anti-human DR5 monoclonal antibody without hepatocyte cytotoxicity. Nat Med 7: 954-960, 2001.

32. Liang X, Liu Y, Zhang Q, et al: Hepatitis B virus sensitizes hepatocytes to TRAIL-induced apoptosis through Bax. J Immunol 178: 503-510, 2007.

33. Mundt B, Wirth T, Zender L, et al: Tumour necrosis factor related apoptosis inducing ligand (TRAIL) induces hepatic steatosis in viral hepatitis and after alcohol intake. Gut 54: 1590-1596, 2005.

34. Mareschi K, Ferrero I, Rustichelli D, et al: Expansion of mesenchymal stem cells isolated from pediatric and adult donor bone marrow. J Cell Biochem 97: 744-754, 2006.

35. Son BR, Marquez-Curtis LA, Kucia M, et al: Migration of bone marrow and cord blood mesenchymal stem cells in vitro is regulated by stromal-derived factor-1-CXCR4 and hepatocyte growth factor-c-met axes and involves matrix metalloproteinases. Stem Cells 24: 1254-1264, 2006. 